\title{
Adaptação Transcultural e Estrutura Fatorial da Versão Brasileira da Escala Right-Wing Authoritarianism
}

\author{
Felipe Vilanova*, 1 \\ Orcid.org/0000-0002-2516-9975 \\ Diogo Araújo DeSousa ${ }^{1,2}$ \\ Orcid.org/0000-0003-4112-6649 \\ Silvia Helena Koller ${ }^{1}$ \\ Orcid.org/0000-0001-9109-6674 \\ AngeloBrandelli Costa ${ }^{3}$ \\ Orcid.org/0000-0002-0742-8152 \\ ${ }^{1}$ Universidade Federal do Rio Grande do Sul, Porto Alegre, RS, Brasil \\ ${ }^{2}$ Universidade Tiradentes, Porto Alegre, RS, Brasil \\ ${ }^{3}$ Pontifícia Universidade Católica do Rio Grande do Sul, Porto Alegre, RS, Brasil
}

\section{Resumo}

A escala mais utilizada para avaliar autoritarismo é a Escala de Autoritarismo de Direita (EAD) originalmente denominada Right-Wing Authoritarianism (RWA). Ela avalia autoritarismo decorrente de crenças associadas à direita política, correlacionando-se com construtos como homofobia, oposição a direitos civis de transexuais, conformidade social e autodeclaração como partidário da direita política. Embora já tenha sido utilizada em contexto nacional assistematicamente, não foram feitos estudos de adaptação para o Brasil. O presente estudo buscou adaptar a EAD para a língua portuguesa e analisar suas propriedades psicométricas no contexto brasileiro através de análise fatorial exploratória. A versão adaptada da EAD apresentou estrutura de quatro fatores com bons índices de consistência interna: Autoritarismo $(\alpha=0,936)$, Constestação à Autoridade $(\alpha=0,858)$, Tradicionalismo $(\alpha=0,871)$ e Submissão à Autoridade $(\alpha=0,897)$. A medida também apresentou validade de critério entre grupos, bem como validade convergente e divergente. Por fim, conclui-se que ela é válida e confiável para investigação de autoritarismo na população brasileira.

Palavras-chave: Autoritarismo, RWA, Right-Wing Authoritarianism, adaptação.

\section{Cross-Cultural Adaptation and Factor Structure of the Brazilian Version of the Right-Wing Authoritarianism}

\begin{abstract}
The most widely used scale to assess authoritarianism is the Right-Wing Authoritarianism (RWA). It assesses authoritarianism related to thoughts commonly associated to the right-wing ideology, and

* Endereço para correspondência: Universidade Federal do Rio Grande do Sul, Rua Ramiro Barcelos, 2600, sala 104, Porto Alegre, RS, Brasil 90.035-003. Fone: (51) 3308-5150. E-mail: felipevilanova2@gmail.com
\end{abstract}


it is correlated to homophobia, opposition to transsexuals' civil rights, social conformity and selfcategorization as a right-wing partisan. Although it has already been used in Brazilian context, there are no studies adapting it to the national context. This study sought to adapt RWA to Brazilian Portuguese and analyze its psychometric properties in the Brazilian context through exploratory factor analysis. The cross-culturally adapted version displayed a four-factor structure and good internal consistency indexes: Authoritarianism $(\alpha=0,936)$, Contestation to Authority $(\alpha=0,858)$, Traditionalism $(\alpha=0,871)$ and Submission to Authority $(\alpha=0,897)$. The instrument also displayed criterion validity between groups, as well as convergent and divergent validity. Therefore, the measure is valid and reliable for the investigation of authoritarianism in the Brazilian population.

Keywords: Authoritarianism, RWA, right-wing authoritarianism, adaptation.

\section{Adaptación Transcultural y Estructura Factorial de la Versión Brasileña de la The Right-Wing Authoritarianism}

\section{Resumen}

El instrumento más utilizado para evaluar el autoritarismo es la Right-Wing Authoritarianism (RWA). El instrumento evalúa el autoritarismo relacionado con las creencias comúnmente asociadas a la ideología derechista, y se correlaciona con la homofobia, la oposición a los derechos civiles de transexuales, la conformidad social y la auto-categorización como un partidario de la derecha política. El instrumento fue utilizado en el contexto brasileño, pero no hay estudios que lo adapten al contexto nacional. Este estudio buscó adaptar la RWA a portugués brasileño y analizar sus propiedades psicométricas en el contexto brasileño mediante análisis factorial exploratorio. La versión culturalmente adaptada ha mostrado una estructura de cuatro factores y ótimos índices de consistencia interna: Autoritarismo ( $\alpha$ $=0,936)$, Contestación a Autoridad $(\alpha=0,858)$, Tradicionalismo $(\alpha=0,871)$ y Sumisión a Autoridad $(\alpha=0,897)$. El instrumento también muestra la validez de criterio entre grupos, así como la validez convergente y divergente. La medida es válida y fiable para la investigación del autoritarismo en la población brasileña.

Palabras clave: Autoritarismo, RWA, right-wing authoritarianism, adaptación.

Após a Segunda Guerra Mundial, houve interesse de sociólogos, filósofos e psicólogos em entender como se deu a ascensão dos regimes totalitários. Diversos estudos foram feitos para explicar como a população forneceu apoio massivo a líderes autoritários (e.g., Adorno, Frenkel-Brinswick, Levinson, \& Sanford, 1950; Altemeyer, 1981; Arendt, 1975), focando em questões como obediência à autoridade (Vilanova, Beria, Costa, \& Koller, 2017; Milgram, 1963). A investigação sistemática do tema autoritarismo com foco no indivíduo a partir da mensuração psicométrica tem suas origens no estudo de Adorno e colaboradores(1950). Os autores avaliaram quais características comporiam a personalidade de alguém mais propenso a apoiar regimes autoritários, o que eles denominaram personalidade autoritária.

Para quantificar a tendência de submissão a líderes autoritários, uma escala denominada F foi construída (Adorno et al., 1950). Em sua forma final, ela é composta por 30 itens que variam de -3 (discordo fortemente) a +3 (concordo fortemente). Embora tenha sido a primeira tentativa de avaliar quantitativamente potenciais autoritários individuais, diversas fragilidades psicométricas foram avaliadas. A escala apresentava itens vagos (e.g. "Familiaridade gera desdém", tradução livre de Adorno et al., 1950, p. 256) ausência de evidências de fidedignidade, e a presença de somente frases afirmativas, o que sujeitaria a escala ao viés de aquiescência (Duckitt, Bizumic, Krauss, \& Heled, 2010; Sibley \& 
Duckitt, 2008).

A Right-Wing Authoritarianism - Escala de Autoritarismo de Direita (EAD; Altemeyer, 1981) surge como alternativa à Escala F. Segundo a base teórica desta escala, a personalidade autoritária é composta pela covariação de três traços: convencionalismo (adesão a normas e valores morais tradicionais); agressão autoritária (agressividade direcionada a várias pessoas, percebida como sancionada por autoridades); e submissão autoritária (submissão acrítica a autoridades estabelecidas). Esses traços compõem o construto autoritarismo decorrente de crenças da direita política, que é avaliado unifatorialmente pela EAD (Altemeyer, 1981, 1996).

Entretanto, também a EAD (Altemeyer, 1981, 1996) apresenta características que comprometem suas propriedades psicométricas. Primeiramente, os escores na EAD se modificam significativamente em situações de ameaça social em relação a situações normais (Altemeyer, 1988; Duckitt \& Fisher, 2003), o que compromete sua conceitualização como uma característica de personalidade. Isto porque é esperado que características de personalidade sejam relativamente estáveis em diferentes situações (Hall, Lindzey, \& Campbell, 2008). Ademais, a maioria dos itens da EAD avalia simultaneamente os traços que compõem a personalidade autoritária, impossibilitando a identificação de se há especificamente um dos traços responsável pela resposta (Funke, 2005). Por exemplo: "Nosso país será ótimo se honrarmos os caminhos dos nossos antepassados [convencionalismo], fizermos o que as autoridades nos dizem [submissão autoritária] e nos livrarmos das 'maçãs podres' que estão estragando tudo [agressão autoritária]" (tradução livre de Altemeyer, 1996, p. 13). Por fim, a conceitualização do autoritarismo como um construto unifatorial pode gerar impasses acerca da identificação de indivíduos que tendam a ser submissos à autoridade, mas não agressivos, e vice-versa (Passini, 2015).

Diante das fragilidades da EAD (Altemeyer, 1981), Duckitt e colaboradores (2010) propuseram modificações ao instrumento. Inicialmente foi proposta uma mudança na definição dos componentes da EAD. Eles deixaram de ser defi- nidos como traços de personalidade e passaram a ser definidos como atitudes sociais independentes por dois motivos: 1) os itens que compõem a EAD consistem em proposições acerca de temáticas sociais; e 2) atitudes sociais são mais variáveis do que traços de personalidade, estando assim em maior consonância com estudos que demonstraram variação significativa dos escores da EAD (e.g., Duckitt \& Fisher, 2003). Cada um dos componentes da EAD abrangeria assim diferentes expressões atitudinais que favoreceriam ou se oporiam à subordinação da liberdade individual à coletividade e a autoridades sociais (Duckitt et al., 2010).

Duckitt e colaboradores (2010) compuseram, portanto, um questionário com itens das versões clássicas da EAD (Altemeyer, 1981, 1988, 1996, 1998), itens da Escala F (Adorno et al., 1950) e de outras escalas de autoritarismo (Cherry \& Byrne, 1977; Kohn, 1974; Lee \& Warr, 1969). Entretanto, os itens da EAD que avaliavam simultaneamente mais de um conjunto de atitudes sociais (anteriormente definidos como traços de personalidade) foram desmembrados, de tal forma que cada item avaliasse apenas um conjunto. Itens que expressavam atitudes homofóbicas também foram excluídos em função da possibilidade de ampliar artificialmente correlações com medidas de preconceito. O questionário conteve 117 itens que foram submetidos a julgamento de experts e sucessivos estudos internacionais para refinar o instrumento.

Uma nova versão da EAD foi então proposta (Duckitt et al., 2010). Ela é composta por 36 itens divididos igualmente entre três fatores independentes: Conservadorismo (tendência a favorecer autoridades institucionais ou grupais de forma submissa e acrítica); Autoritarismo (tendência a apoiar o uso de métodos coercitivos de controle social, como pena de morte); e Tradicionalismo (adesão e apoio a valores morais tradicionais). A estrutura fatorial da versão proposta está em consonância com estudos que demonstram melhor adequação dos dados empíricos obtidos com a EAD a um modelo trifatorial ao invés de unifatorial (Funke, 2005; Mavor, Louis, \& Sibley, 2010).

Desde sua construção, a EAD já foi utilizada 
em diversos estudos. Os escores nesta escala estão diretamente associados a escores de homofobia (Adams, Nagoshi, Filip-Crawford, Terrell, \& Nagoshi, 2016; Crawford, Brandt, Inbar, \& Mallinas, 2016; Goodnight, Cook, Parrott, \& Peterson, 2014; Sousa, 2016; Stones, 2006); conformidade social (Duckitt, Wagner, du Plessis, \& Birum, 2002); atitudes favoráveis à tortura (Benjamin, 2016); autodeclaração como partidário da direita política (Passini, 2015); baixos níveis de escolaridade (Carvacho et al., 2013); e oposição a direitos civis de transsexuais (Tee \& Hegarty, 2006). A relevância dos temas com os quais a escala pode ser relacionada é patente, especialmente considerando o cenário político brasileiro atual, o qual pode ser melhor entendido à luz dos estudos sobre autoritarismo. Todavia, a EAD tende a apresentar padrões diferentes de correlações entre as culturas (Duckitt et al., 2010), sendo fundamental a análise de suas propriedades psicométricas em diferentes contextos.

O presente estudo é o primeiro que visa adaptar e validar a versão da EAD proposta por Duckitt e colaboradores (2010) em um contexto diferente dos utilizados para a criação da escala. Outras versões da EAD já foram adaptadas para diferentes contextos, como por exemplo para a Argentina (Etchezahar, 2012), para a África do Sul (Duckitt, 1993) e para a Turquia (Güldü, 2011). Enquanto as versões adaptadas para a Argentina (Etchezahar, 2012) e para a Turquia (Güldü, 2011) apresentaram estrutura bifatorial, a versão adaptada para a África do Sul (Duckitt, 1993) apresentou estrutura unifatorial. Em estudo recente, a EAD adaptada para a África do Sul (Duckitt, 1993) teve suas propriedades psicométricas reavaliadas e se verificou que ela apresentou estrutura trifatorial com índices inadequados de consistência interna (Gray \& Durrheim, 2006). Portanto, há uma variação intercultural e temporal na estrutura fatorial das diferentes escalas que avaliam autoritarismo que aponta para influência da cultura no construto autoritarismo e realça a necessidade de avaliação das propriedades psicométricas do instrumento em diferentes contextos.

Embora ainda não tenha sido feito um estudo de adaptação da EAD para o Brasil, ela já foi utilizada em contexto nacional de maneira assistemática (e.g., Barros, Torres, \& Pereira, 2009; Cantal, Milfont, Wilson, \& Gouveia, 2015; Guimarães, Torres, \& de Faria, 2005; Santos, 2015; Torres, de Faria, Guimarães, \& Martignoni, 2007; Vilela, 2012) tanto em sua forma clássica (Altemeyer, 1988) quanto em sua forma alternativa (Duckitt et al., 2010). Portanto, a fim de aprimorar a precisão de resultados obtidos nacionalmente, o objetivo do presente estudo foi adaptar a EAD proposta por Duckitt e colaboradores (2010) para a língua portuguesa e analisar suas propriedades psicométricas no contexto brasileiro. As propriedades psicométricas investigadas incluíram: estrutura fatorial da escala adaptada ao Brasil; consistência interna; e evidências de validade de critério entre grupos políticos e com escores de preconceito contra diversidade sexual e de gênero.

\section{Método}

\section{Participantes}

Participaram do estudo 518 indivíduos com idades entre 18 e 79 anos $(M=39,31 ; D P$ $=17,93), 59,8 \%$ do sexo masculino. Destes, 307 (59,4\%) moram na região sul, $184(35,6 \%)$ moram na região sudeste, $18(3,5 \%)$ moram na região nordeste e $8(1,5 \%)$ moram na região centro-oeste. A Tabela 1 apresenta a caracterização sociodemográfica da amostra em termos de etnia, escolaridade, classe socioeconômica e religião.

\section{Procedimentos}

Após a autorização concedida pelo autor da escala original, iniciou-se o processo de adaptação transcultural da Right-Wing Authoritarianism (EAD; Duckitt et al., 2010). A tradução inglês-português foi conduzida independentemente por dois pesquisadores experts em psicologia social, brasileiros nativos e fluentes em inglês. Em seguida, dois experts em avaliação psicológica avaliaram os itens traduzidos e sugeriram mudanças. Posteriormente foi solicitado a três voluntários que se autodenominaram "militantes" da direita política que escolhessem a me- 
Tabela 1

Caracterização Sociodemográfica da Amostra

Característica

$n(\%)$

Total $(N=518)$

Raça / Etnia $n(\%)$

Branca

$449(86,7)$

Parda

$46(8,9)$

Negra

Indígena

$3(0,6)$

Amarela

$2(0,4)$

Classe Econômica $n(\%)$

Classe A - renda domiciliar mensal igual ou maior a 20 salários mínimos

Classe B - renda domiciliar mensal de 10 a 20 salários mínimos

$101(19,5)$

Classe C - renda domiciliar mensal de 4 a 10 salários mínimos

Classe D - renda domiciliar mensal de 2 a 4 salários mínimos

$120(23,2)$

Classe E - renda domiciliar mensal de até 2 salários mínimos

Não sei

Nível de Escolaridade $n(\%)$

Fundamental incompleto ( $1^{\circ}$ grau $)$

Fundamental completo ( $1^{\circ}$ grau $)$

Médio incompleto ( $2^{\circ}$ grau $)$

Médio completo $\left(2^{\circ}\right.$ grau $)$

Superior incompleto (universitário)

Superior completo (universitário)

Pós Graduação

Autoidentificação política $n(\%)$

Esquerda

$166(32)$

Centro-esquerda

Centro

Centro-direita

Direita

Nenhuma das acima citadas

$69(13,3)$

lhor proposta de adaptação do instrumento, havendo a possibilidade de sugerir uma alternativa às propostas. Os itens resultantes foram então retrotraduzidos por um dos pesquisadores que realizou a primeira etapa da tradução e enviados ao autor da escala original. Em todas as etapas foram levados em consideração os aspectos relevantes para a adaptação transcultural de instrumentos psicológicos (Borsa, Damásio, \& Bandeira, 2012), tais como equivalência conceitual e idiomática. O método de avaliação da validade de conteúdo utilizado em todas as etapas foi o 
consenso entre os avaliadores ou os experts.

A coleta de dados com a versão brasileira da escala foi realizada por meio de formulário onli$n e$. Os participantes foram convidados a responder através de um link de divulgação postado em rede social entre outubro e novembro de 2016. Um anúncio de divulgação da pesquisa foi gerado via rede social para que um maior número de pessoas fosse alcançado. Antes de responder às perguntas do questionário, os indivíduos expressaram sua concordância por meio de Termo de Consentimento Livre e Esclarecido. O anonimato foi garantido e somente os pesquisadores tiveram acesso aos dados, conforme considerações éticas da Resolução n. 510/2016 do Conselho Nacional de Saúde para pesquisas com seres humanos. A amostra foi recrutada por conveniência. O delineamento do presente estudo foi aprovado pelo Comitê de Ética em Pesquisa da universidade a que o projeto está vinculado.

\section{Instrumentos}

O instrumento completo foi composto por um questionário sociodemográfico investigando as seguintes variáveis dos participantes: estado e cidade em que reside, gênero, idade, raça/etnia, estado civil, classe socioeconômica, nível de escolaridade, em qual tipo de escola (pública ou privada) estudou durante a maior parte da vida, se já estudou em universidade (em caso afirmativo, se era pública ou particular) se possui crença religiosa ou espiritual e qual, e qual a frequência de envolvimento com atividades religiosas. Posteriormente foi perguntado se o indivíduo é filiado a algum partido político (e qual) e em qual parte do espectro político (centro-esquerda, esquerda, centro, centro-direita, direita ou nenhuma) ele se situaria. Em seguida, os participantes respondiam a versão brasileira da EAD (Duckitt et al., 2010) e, por último, a Escala Revisada de Preconceito contra Diversidade Sexual e de Gênero (Costa, Machado, Bandeira, \& Nardi, 2016).

Right-Wing Authoritarianism. Conforme descrito detalhadamente na seção de introdução, a Right-Wing Authoritarianism (Duckitt et al., 2010) é uma medida de autorrelato composta por três fatores com 12 itens cada: (1) autoritarismo;
(2) conservadorismo; (3) tradicionalismo. Em seu estudo de desenvolvimento, ela apresentou bons índices de ajuste quanto à estrutura de três fatores $(\mathrm{RMSEA}=0,061 ; \mathrm{SRMR}=0,065 ; \mathrm{GFI}=$ 0,95; CFI = 0,95; Duckitt et al., 2010). Na versão em português, cuja adaptação dos itens é descrita na seção de resultados, as respostas são dadas em uma escala de concordância de 5 pontos, variando de 1 (discordo totalmente) a 5 (concordo totalmente).

Escala Revisada de Preconceito contra Diversidade Sexual e de Gênero. A Escala Revisada de Preconceito contra Diversidade Sexual e de Gênero (Costa, Bandeira, \& Nardi, 2015; Costa et al., 2016) é uma medida de autorrelato unidimensional composta por 18 itens. As propriedades psicométricas da escala foram avaliadas através de TRI (modelo da família Rasch), análise fatorial confirmatória (AFC) e validade de critério. A análise de contrastes principais mostrou a presença de um componente eigenvalue $=22,4$, explicando $55,5 \%$ da variância dos itens. Além disso, o modelo unifatorial apresentou bons ajustes na AFC $\left(\mathrm{X}^{2} / g l=43,02, p<\right.$ $0,001, \mathrm{CFI}=0,96, \mathrm{TLI}=0,96$, RMSEA 0,07) e a escala diferenciou de maneira satisfatória grupos que historicamente tem diferido em diversas medidas de preconceito. Na presente amostra o instrumento apresentou boa consistência interna ( $\alpha$ de Cronbach $=0,94)$ e os itens variam de 1 (discordo totalmente) a 5 (concordo totalmente).

\section{Análise de Dados}

A versão brasileira da EAD foi submetida a uma análise fatorial exploratória para investigação de sua estrutura fatorial no novo contexto (Damásio, 2012) e a uma análise paralela, a fim de se obter eigenvalues através de permutação aleatória dos dados. Primeiramente, dois métodos de avaliação foram utilizados para observar a adequação da matriz de dados à fatoração: o critério de Kaiser-Meyer-Olkin (KMO) e o teste de esfericidade de Bartlett. Em seguida, a AFE foi conduzida a partir de uma fatoração por eixos principais (principal axis factoring), com rotação oblíqua oblimin. O número de fatores retidos foi delimitado a partir do critério de Kaiser-Guttman (i.e., eigenvalue $>1$ ) e da apresentação de 
eigenvalues resultantes da AFE superiores aos da análise paralela (O'Connor, 2000). Cargas fatoriais acima de 0,40 foram consideradas adequadas para retenção dos itens nos fatores.

Para investigação de consistência interna, foram calculados alfas de Cronbach do escore total da EAD e de suas subescalas. Para investigação de evidências de validade de critério entre grupos, foram realizadas Análises de Variância (ANOVAs) entre participantes que se identificaram em diferentes pontos de um espectro político (esquerda; centro-esquerda; centro; centro-direita; direita; nenhum destes). Por fim, para investigação de evidências de validade de critério convergente, foram realizadas correlações de Pearson entre os escores da EAD e os escores da Escala Revisada de Preconceito contra Diversidade Sexual e de Gênero (Costa et al., 2016).

\section{Resultados}

\section{Adaptação Transcultural}

Com base nas sugestões dos experts em avaliação psicológica, alguns itens da EAD com mais de um objeto sintático ou sujeito foram desmembrados a fim de que houvesse apenas um destes por item. Assim, o item da escala original "our country will be great if we show respect for authority and obey our leaders" foi desmembrado em "nosso país será melhor se mostrarmos respeito à autoridade" e "nosso país será melhor se obedecermos nossos líderes". O item original "people should be ready to protest against and challenge laws they don't agree with" foi desmembrado em "as pessoas devem estar prontas para protestar contra leis com as quais elas não concordam" e "as pessoas devem estar prontas para desafiar leis com as quais elas não concordam". O item "the more people there are that are prepared to criticize the authorities, challenge and protest against the government, the better it is for society" foi desmembrado em "quanto maior o número de pessoas preparadas para criticar as autoridades, melhor para a sociedade", "quanto maior o número de pessoas preparadas para desafiar o governo, melhor para a sociedade" e "quanto maior o número de pessoas preparadas para protestar contra o gover- no, melhor para a sociedade". O item "the real keys to the 'good life' are respect for authority and obedience to those who are in charge" foi desmembrado em "o segredo para uma boa vida é o respeito pela autoridade" e "o segredo para uma boa vida é a obediência àqueles que estão no controle". O item "everyone should have their own lifestyle, religious beliefs, and sexual preferences, even if it makes them different from everyone else" foi desmembrado em "as pessoas deveriam ter os seus próprios estilos de vida mesmo se isso torná-las diferentes do resto da sociedade", "as pessoas deveriam ter as suas próprias preferências sexuais mesmo se isso torná-las diferentes do resto da sociedade" e "as pessoas deveriam ter as suas próprias crenças religiosas mesmo se isso torná-las diferentes do resto da sociedade". Por último, o item "people who say our laws should be enforced more strictly and harshly are wrong. We need greater tolerance and more lenient treatment for lawbreakers" foi desmembrado em "as pessoas que dizem que nossas leis deveriam ser aplicadas de maneira mais rigorosa estão erradas" e "nós precisamos de maior tolerância e mais leniência no tratamento de infratores".

A versão adaptada da escala utilizada na coleta de dados para investigação de propriedades psicométricas, portanto, contou com 44 itens, oito a mais que a escala original, por conta dos desmembramentos descritos. Essa versão foi submetida à avaliação dos voluntários que se autodenominaram "militantes" da direita, para pré-teste, os quais não sugeriram alterações nos itens. A retrotradução dessa versão, enviada ao autor original da escala, foi aprovada por ele sem indicação de modificações.

\section{Estrutura Fatorial}

$\mathrm{Na}$ avaliação preliminar de adequação da matriz de dados, o índice de KMO foi 0,964, considerado ótimo. Os resultados do teste de esfericidade de Bartlett também indicaram adequação para fatoração $(p<0,001)$. Os resultados da extração de fatores na AFE indicaram uma solução de cinco fatores. Os eigenvalues resultantes da AFE foram respectivamente 17,51; 2,$40 ; 1,73 ; 1,50$ e 0,911 . O quinto fator violou 
o critério de Kaiser-Guttman e foi o único que teve eigenvalue resultante da AFE inferior ao gerado pela análise paralela (eigenvalue gerado pela análise paralela para o fator $1=1,605$; para o fator $2=1,539$; para o fator $3=1,492$; para o fator $4=1,450$; para o fator $5=1,413$ ). Portanto, a solução resultante foi de quatro fatores, respectivamente: Autoritarismo (AT), Contestação à Autoridade (CA), Tradicionalismo (TR) e Submissão à Autoridade (SA). Os quatro fatores retidos explicaram cumulativamente $52,69 \%$ da variância da escala (Tabela 2).

Dez itens não alcançaram cargas fatoriais acima de 0,40 em nenhum dos quatro fatores retidos: "As pessoas deveriam parar de ensinar crianças a obedecer à autoridade"; "O país irá prosperar se os jovens pararem de experimentar drogas, álcool e sexo, e prestarem mais atenção aos valores de família"; "Um governo forte e rígido vai prejudicar nosso país e não o ajudar"; "Os tribunais estão certos em serem mais brandos com traficantes de drogas. Punição não adiantaria de nada em casos como esses"; "As pessoas devem ser permitidas a fazer discursos e escrever livros incitando a derrubada do governo"; "As pessoas devem estar prontas para protestar contra leis com as quais elas não concordam"; "A mídia radical está envenenando as mentes dos nossos jovens"; "É importante que preservemos nossos valores tradicionais e padrões morais"; "Os novos estilos de vida e comportamentos radicais e pecaminosos de muitos jovens poderá destruir nossa sociedade"; e "Os modos e valores tradicionais ainda mostram a melhor forma de se viver" (Tabela 2).

A partir da análise do conteúdo dos itens dos quatro fatores, é possível notar que o primeiro fator agrupou itens relacionados à tendência a retirar liberdades civis e apoiar medidas punitivas severas, sendo denominado 'Autoritarismo'. O segundo fator agrupou itens associados à tendência a criticar, desafiar e protestar contra autoridade, sendo denominado 'Contestação à Autoridade'. O terceiro fator agrupou itens associados a valores e padrões morais tradicionais, sendo denominado 'Tradicionalismo'. Já o quarto fator agrupou itens relacionados à propensão a obedecer e respeitar autoridades, sendo deno- minado 'Submissão à Autoridade'.

Os escores dos fatores foram calculados a partir da média aritmética simples dos escores dos itens que compõem cada fator. As respostas aos itens que apresentaram carga fatorial negativa nos fatores 'Autoritarismo', 'Contestação à Autoridade' e 'Submissão à Autoridade' foram recodificadas para inverter seus valores para o cálculo dos escores desses fatores (i.e., respostas 1 na escala foram recodificadas como 5 , e vice-versa, e respostas 2 na escala foram recodificadas como 4, e vice-versa). Para o fator 'Tradicionalismo', as respostas a todos os itens foram recodificadas para inverter seus valores a fim de indicar que, quanto maior o escore, maior o nível de tradicionalismo do respondente.

Para o cálculo do escore geral da EAD, as respostas a todos os itens do fator 'Contestação à Autoridade' foram recodificados para inverter seus valores antes de se calcular o escore deste fator. Em seguida, o escore geral da escala foi calculado a partir da média aritmética simples dos escores dos quatro fatores. Assim, quanto maior o escore geral do indivíduo na EAD, maior seu nível de atitudes sociais autoritárias, tradicionalistas e maior sua tendência a se submeter à autoridade.

$\mathrm{O}$ alfa de Cronbach do escore geral da EAD demonstrou alta consistência interna $(\alpha=0,957$; IC $95 \%[0,952 ; 0,962])$. De forma similar, os coeficientes dos escores dos fatores também foram altos: 'Autoritarismo' $(\alpha=0,936$; IC 95\% [0,928; $0,944])$; 'Contestação à Autoridade' $(\alpha=0,858$; IC 95\% [0,840; 0,875]); 'Tradicionalismo' $(\alpha=$ 0,871 ; IC 95\% [0,855; 0,886]); e 'Submissão à Autoridade' ( $\alpha=0,897$; IC 95\% [0,884; 0,909]).

\section{Evidências de Validade de Critério entre Grupos}

Como evidência de validade de critério entre grupos, a Figura 1 apresenta os resultados para os escores da EAD entre os grupos de pessoas que se identificaram em diferentes pontos de um espectro político composto por esquerda, centro-esquerda, centro, centro-direita, direita e "sem identificação".

Pode-se perceber que, para o fator 'Autoritarismo', os escores foram progressivamente mais 
da Escala Right-Wing Authoritarianism.

Tabela 2

Fatores e Cargas Fatoriais da Análise Fatorial Exploratória da Versão Brasileira da EAD

\begin{tabular}{|c|c|c|c|c|}
\hline Item & AT & CA & TR & SA \\
\hline Do jeito que as coisas estão indo nesse país, serão ... & 0,770 & 0,020 & 0,005 & 0,157 \\
\hline A situação do nosso país está ficando tão séria que ... & 0,770 & 0,015 & $-0,045$ & 0,122 \\
\hline Ser gentil com criminosos só os encoraja a tirar ... & 0,751 & 0,005 & $-0,024$ & 0,076 \\
\hline A pena de morte é bárbara e nunca justificável. & $-0,710$ & 0,057 & 0,032 & 0,096 \\
\hline Os crimes e as desordens públicas recentes mostram ... & 0,710 & $-0,084$ & $-0,030$ & 0,123 \\
\hline O que o nosso país realmente precisa é uma dose ... & 0,684 & 0,018 & $-0,071$ & 0,189 \\
\hline Nós deveríamos esmagar todos os elementos ... & 0,671 & 0,121 & $-0,006$ & 0,162 \\
\hline Nossas prisões são um desastre. Ao invés de tanta ... & $-0,640$ & 0,132 & 0,037 & 0,052 \\
\hline Nós precisamos de maior tolerância e mais leniência ... & $-0,633$ & 0,177 & 0,034 & 0,137 \\
\hline As pessoas que dizem que nossas leis deveriam ser ... & $-0,582$ & 0,098 & 0,007 & 0,006 \\
\hline Nossa sociedade NÃO precisa de um governo mais ... & $-0,566$ & 0,158 & 0,022 & $-0,051$ \\
\hline O país irá prosperar se os jovens pararem de ... & 0,388 & 0,122 & $-0,307$ & 0,255 \\
\hline Os tribunais estão certos em serem mais brandos ... & $-0,381$ & 0,074 & 0,038 & $-0,058$ \\
\hline Quanto maior o número de pessoas preparadas para ... & 0,046 & 0,829 & $-0,064$ & $-0,051$ \\
\hline Quanto maior o número de pessoas preparadas ... & 0,022 & 0,760 & 0,035 & 0,028 \\
\hline Quanto maior o número de pessoas ... & $-0,012$ & 0,684 & 0,057 & $-0,102$ \\
\hline As pessoas devem estar prontas para desafiar leis ... & $-0,032$ & 0,623 & $-0,049$ & $-0,061$ \\
\hline Estudantes de colégios e universidades devem ... & $-0,340$ & 0,464 & 0,130 & $-0,007$ \\
\hline É ótimo que atualmente muitos jovens estejam ... & $-0,236$ & 0,420 & 0,203 & $-0,023$ \\
\hline As pessoas devem ser permitidas a fazer discursos ... & $-0,014$ & 0,391 & $-0,044$ & $-0,201$ \\
\hline As pessoas devem estar prontas para protestar ... & $-0,171$ & 0,373 & 0,109 & 0,043 \\
\hline As pessoas deveriam parar de ensinar crianças ... & $-0,116$ & 0,339 & 0,084 & $-0,026$ \\
\hline Um governo forte e rígido vai prejudicar nosso ... & $-0,127$ & 0,304 & 0,028 & $-0,206$ \\
\hline As pessoas deveriam ter as suas próprias ... & $-0,024$ & $-0,012$ & 0,815 & $-0,058$ \\
\hline Não há nada de errado com sexo antes do casamento. & 0,118 & $-0,050$ & 0,711 & $-0,058$ \\
\hline Não tem absolutamente nada errado com praias ... & $-0,033$ & $-0,060$ & 0,671 & $-0,015$ \\
\hline As pessoas deveriam ter os seus próprios estilos ... & $-0,093$ & 0,041 & 0,654 & $-0,091$ \\
\hline As pessoas deveriam ter as suas próprias crenças ... & $-0,047$ & 0,077 & 0,644 & $-0,019$ \\
\hline As pessoas deveriam prestar menos atenção à ... & 0,039 & 0,118 & $\mathbf{0 , 5 2 7}$ & $-0,013$ \\
\hline As leis de Deus sobre aborto, pornografia e ... & 0,116 & 0,097 & $-0,509$ & 0,328 \\
\hline Ninguém deveria se restringir ao que é ... & $-0,105$ & 0,253 & 0,446 & 0,094 \\
\hline Tem muita coisa de errado com os valores ... & $-0,112$ & 0,222 & 0,409 & 0,047 \\
\hline Os novos estilos de vida e comportamentos radicais ... & 0,315 & 0,094 & $-0,385$ & 0,290 \\
\hline Os modos e valores tradicionais ainda mostram a ... & 0,347 & $-0,049$ & $-0,376$ & 0,180 \\
\hline A mídia radical está envenenando as mentes dos ... & 0,205 & 0,046 & $-0,246$ & 0,226 \\
\hline O segredo para uma boa vida é a obediência ... &,- 010 & $-0,042$ & $-0,021$ & 0,742 \\
\hline
\end{tabular}


As autoridades devem ser obedecidas porque elas ...

Nosso país será melhor se obedecermos nossos líderes.

O segredo para uma boa vida é o respeito ...

O que nosso país mais precisa é disciplina, com ...

Nossos líderes deveriam ser obedecidos sem ...

As virtudes mais importantes que as crianças devem ...

Nosso país será melhor se mostrarmos respeito à ...

É importante que preservemos nossos valores ...

Variância explicada \%

$\begin{array}{crrr}-0,046 & -0,093 & -0,044 & \mathbf{0 , 7 3 6} \\ 0,005 & -0,091 & -0,009 & \mathbf{0 , 6 9 0} \\ 0,113 & -0,037 & -0,074 & \mathbf{0 , 6 2 8} \\ 0,203 & -0,006 & -0,037 & \mathbf{0 , 5 5 9} \\ -0,036 & -0,081 & -0,085 & \mathbf{0 , 5 4 8} \\ 0,339 & 0,087 & -0,039 & \mathbf{0 , 5 3 2} \\ 0,234 & -0,147 & -0,009 & \mathbf{0 , 4 9 3} \\ 0,275 & -0,045 & -0,342 & 0,222 \\ 39,876 & 5,462 & 3,934 & 3,419\end{array}$

Nota. Cargas fatoriais acima de 0,40 em negrito.

altos no espectro esquerda-direita, sendo que, quanto mais à direita, maior a pontuação neste fator $\left[F(5,512)=155,45, p<0,001, \eta^{2}=0,60\right]$. Testes post-hoc Bonferroni foram conduzidos a fim de avaliar quais grupos se diferenciavam significativamente. Houve diferenças significativas entre todos os grupos $(p<0,05)$ exceto entre os que se declararam no centro do espectro político e os que declararam não estar em nenhuma parte do espectro. Para o fator 'Contestação à Autoridade', os escores foram progressivamente menores no espectro esquerda-direita $[F(5,512)=$ $\left.63,65, p<0,001, \eta^{2}=0,38\right]$. De acordo com testes post-hoc Bonferroni, somente não houve diferença significativa entre direita e centro-direita. Para o fator 'Tradicionalismo', os escores foram progressivamente maiores no espectro esquerda-direita $\left[F(5,512)=75,76, p<0,001, \eta^{2}=0,42\right]$. Os testes post-hoc Bonferroni demonstraram que não houve diferença significativa somente entre esquerda e centro-esquerda, entre centro e aqueles que não se enquadraram no espectro político, e entre centro-direita e aqueles que não se enquadraram no espectro político. Por fim, para o fator 'Submissão à Autoridade, os escores também foram progressivamente mais altos no espectro esquerda-direita $[F(5,512)=53,20, p$ $\left.<0,001, \eta^{2}=0,34\right]$. Pelos testes post-hoc Bonferroni, não houve diferenças significativas somente entre esquerda e centro-esquerda, entre centro e centro-direita e entre centro e os que não se classificaram no espectro político.

Considerando o escore geral da EAD, também foi progressivamente maior no espectro es- querda-direita $[F(5,512)=167,841, p<0,001$, $\left.\eta^{2}=0,62\right]$. De acordo com testes post-hoc Bonferroni, somente não houve diferenças significativas entre esquerda e centro-esquerda, entre centro e os que não se classificaram no espectro político, e entre centro-direita e os que não se classificaram no espectro político.

Como evidência de validade de critério convergente, foram encontradas correlações positivas significativas entre o escore da Escala Revisada de Preconceito contra Diversidade Sexual e de Gênero e o escore geral da EAD ( $r=0,746$; $p<0,001)$, e os escores dos fatores 'Autoritarismo' ( $r=0,633 ; p<0,001)$; 'Tradicionalismo' ( $r$ $=0,784 ; p<0,001)$ e 'Submissão à Autoridade' $(r=0,639 ; p<0,001)$. Além disso, foi encontrada uma correlação negativa significativa entre o escore da Escala Revisada de Preconceito contra Diversidade Sexual e de Gênero e o escore do fator 'Contestação à Autoridade' ( $r=-0,441 ; p$ $<0,001)$.

\section{Discussão}

O presente estudo descreveu o processo de adaptação transcultural da escala Right-Wing Authoritarianism (EAD; Duckitt et al., 2010) para o Brasil. Os índices de consistência interna (alfas de Cronbach) apresentados foram superiores aos de estudos anteriores que utilizaram diferentes versões da EAD no Brasil (Barros et al., 2009; Guimarães et al., 2005; Torres et al., 2007; Vilela, 2012). Além disso, a solução fatorial proposta explicou uma maior parte da variância dos 


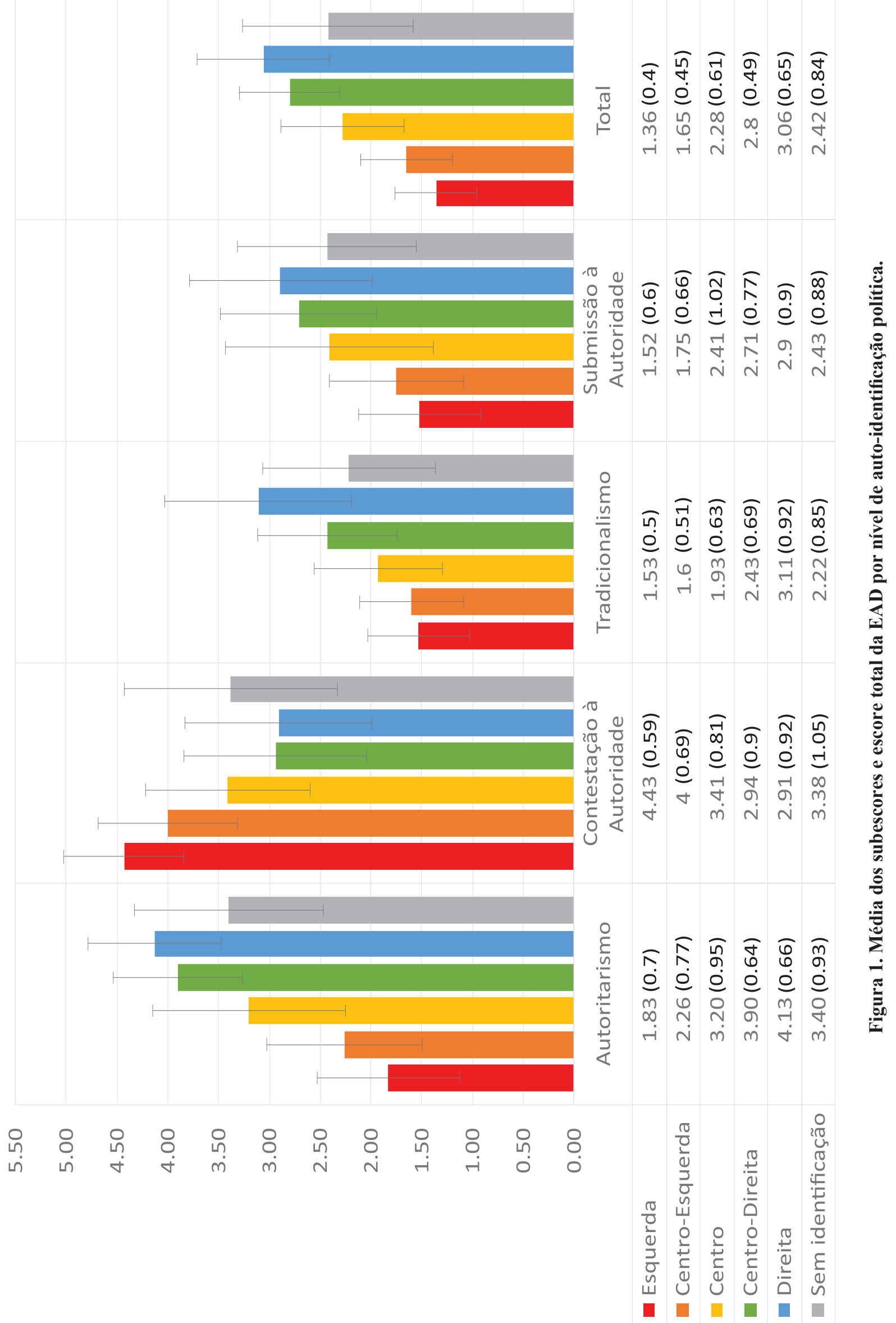


escores da escala do que apresenta em estudos anteriores (Barros et al., 2009; Guimarães et al., 2005; Torres et al., 2007).

Alguns itens propostos nesta adaptação cultural não apresentaram cargas fatoriais adequadas em nenhum dos fatores. Um deles foi um item desmembrado em função de apresentar originalmente mais de um sujeito ou objeto: “As pessoas devem estar prontas para protestar contra leis com as quais elas não concordam". Em contrapartida, outro item análogo que também foi desmembrado foi retido: "As pessoas devem estar prontas para desafiar leis com as quais elas não concordam". Neste caso, percebe-se que a ideia de 'desafio', do item retido, foi mais forte que 'protesto' do item eliminado. O item "Os tribunais estão certos em serem mais brandos com traficantes de drogas. Punição não adiantaria de nada em casos como esses" também foi excluído da escala por não ter apresentado carga fatorial adequada. A duplicidade de informação pode ter prejudicado a compreensão. Os experts não sugeriram divisão, no entendimento de que se trataria de uma mesma informação nas duas frases, no entanto, a compreensão pode ter sido prejudicada, como em um caso em que o sujeito concorde com penas mais brandas, mas discorde da eficácia da punição.

Dois itens relacionados à ideia de valores também foram excluídos: "Os modos e valores tradicionais ainda mostram a melhor forma de se viver" e "É importante que preservemos nossos valores tradicionais e padrões morais". A ideia de valores tradicionais talvez não seja compreendida completamente no contexto brasileiro. Um único item que menciona valores permaneceu na adaptação: "Tem muita coisa de errado com os valores, os costumes e a moralidade tradicionais". É possível que a compreensão tenha sido potencializada porque o item contextualiza o que seriam valores mencionando costumes e moralidade tradicionais. Futuras versões da EAD podem testar modificações na redação dos itens originais relacionados a valores utilizando a ideia de costume e moralidade tradicionais.

Outros dois itens excluídos tratavam do comportamento de jovens, sexual e de uso de substância: "O país irá prosperar se os jovens pararem de experimentar drogas, álcool e sexo, e prestarem mais atenção aos valores de família"; e "Os novos estilos de vida e comportamentos radicais e pecaminosos de muitos jovens poderá destruir nossa sociedade". Os itens mencionam sexualidade e consumo de álcool, que apresentam características distintas no Brasil em comparação com o contexto anglo-saxão. Por exemplo, no contexto Brasileiro nunca foram propostas políticas de abstinência sexual para o controle de infecções sexualmente transmissíveis como aconteceu em outros países (Santelli et al., 2006). Embora setores do congresso nacional tenham discutido propostas similares recentemente (Carrara, 2012), é possível que a representação social de uma atitude autoritária relacionada à moralidade sexual seja inerte do ponto de vista da população. O mesmo em relação ao consumo de álcool, o qual entre jovens chega a $81,7 \%$ (Cerutti, Ramos, \& Argimon, 2015).

O item "As pessoas devem ser permitidas a fazer discursos e escrever livros incitando a derrubada do governo" e o item "A mídia radical está envenenando as mentes dos nossos jovens" também foram excluídos da versão final brasileira. Em relação ao primeiro, hipotetiza-se que o baixo nível de leitura do Brasil afasta a compreensão direta em escrita de livros como veículo para a ação política, especialmente no contexto político autoritário [ $44 \%$ da população brasileira não lê e 30\% nunca comprou um livro (Failla, 2016)]. Em relação ao segundo item, a versão original mencionava a ideia de "trashy magazines and radical literature" e a tentativa de adaptação tentou aproximar o contexto brasileiro do significado original do item, provavelmente sem sucesso. Possivelmente os experts e aqueles que se declararam militantes de direita sugeriram a permanência do item sem modificações por estarem imersos em um contexto em que o jargão político utilizando essa expressão (mídia radical) seja mais prevalente. No entanto, os resultados do estudo sugerem que a população investigada pode não compreender esse tipo de vocabulário. Talvez não exista no Brasil a ideia de mídia radical conectada a uma visão política autoritária.

Por fim, o item "Um governo forte e rígido 
vai prejudicar nosso país e não o ajudar" e o item "As pessoas deveriam parar de ensinar crianças a obedecer à autoridade" também foram excluídos. No Brasil temos a prevalência de duas visões que contrastam com a visão do norte global de onde essas escalas são oriundas. A primeira delas é a visão liberal conservadora que congrega pessoas que defendem o estado mínimo do ponto de vista da regulação econômica e social ao mesmo tempo que defendem uma posição conservadora em relação aos comportamentos (Datafolha, 2014). A segunda visão pode dizer respeito à esquerda autoritária, ou seja, posições de esquerda que defendem não só a regulamentação estatal, mas também a submissão a líderes autoritários (de Regt, Mortelmans, \& Smits, 2011). Provavelmente, este tipo de distribuição fez com que esses itens não estivessem em harmonia com o restante da escala.

Percebe-se que o fator originalmente denominado 'Conservadorismo' foi subdivido a partir dos resultados da amostra brasileira. Os itens que originalmente apresentaram carga negativa neste fator se aglutinaram em um novo fator no presente estudo. Por estar relacionado a ideias de criticar, desafiar e protestar contra autoridade, foi denominado 'Contestação à Autoridade'. A literatura já descreveu situação similar (Mavor et al., 2010), entretanto outros autores preferiram manter a estrutura de três fatores via análise fatorial confirmatória, uma vez que ela também apresentava bons índices psicométricos. No atual estudo foi decidido manter a estrutura resultante da AFE vista a limitação de tamanho de amostra para testar os dois modelos concorrentes em uma análise fatorial confirmatória (i.e., o modelo original de três fatores e o modelo empírico proposto na AFE deste estudo). Ademais, o termo 'Conservadorismo' foi modificado para 'Submissão à Autoridade'. Isto porque, embora o respeito e a obediência à autoridade sejam parte da doutrina conservadora (Coutinho, 2014; Scruton, 2015), esta é muito mais abrangente. Assim, utilizar o termo 'Conservadorismo' tem menor precisão conceitual do que o termo 'Submissão à Autoridade'.

A estrutura fatorial obtida também é diferente de estudos anteriores com outras versões da EAD (e.g. Etchezahar, 2012). Tendo em vista que o autoritarismo é conceituado como uma atitude social (Duckitt et al., 2010) é plausível que os instrumentos que o avaliem variem conforme a sociedade muda, como já evidenciado pela literatura (Gray \& Durrheim, 2006). Logo, é fundamental a reavaliação das propriedades psicométricas da EAD periodicamente, assim como em diferentes populações, as quais podem apresentar características sociodemográficas diferentes da amostra do presente estudo e consequentemente manifestar o autoritarismo de diferentes formas.

A média geral da escala, bem como a média dos subfatores 'Autoritarismo', 'Tradicionalismo' e 'Submissão à Autoridade', foram sequencialmente maiores entre os participantes que se autodeclararam no espectro esquerda-direita, demonstrando boa validade de critério, uma vez que a escala avalia atitudes sociais decorrentes de crenças relacionadas à direita política (Passini, 2015). No fator 'Contestação à Autoridade', a média no espectro esquerda-direita foi sequencialmente menor, o que também reforça a validade de critério.

Finalmente, a média dos escores na Escala de Preconceito Contra Diversidade Sexual e de Gênero se correlacionaram positivamente com a média dos escores nos fatores 'Tradicionalismo', 'Autoritarismo' e 'Submissão à Autoridade', e negativamente com a média dos escores no fator 'Contestação à Autoridade'. Este resultado era esperado uma vez que a literatura demonstra relação robusta direta entre autoritarismo e homofobia (Adams et al., 2016; Goodnight et al., 2014; Sousa, 2016; Stones, 2006), bem como entre autoritarismo e oposição a direitos civis de transsexuais (Tee \& Hegarty, 2006).

O presente estudo apresenta limitações que devem ser levadas em consideração. Primeiro, o espectro político avaliado é muito abrangente e não especifica subdivisões dos posicionamentos políticos, como liberais, conservadores, anarquistas, dentre outros. A fim de investigar melhor como se dá o fenômeno do autoritarismo no contexto brasileiro, estudos futuros poderão utilizar outras formas de categorização política. Segundo, parcela considerável da amostra se 
declarou de etnia branca, não correspondendo à diversidade étnica da sociedade brasileira. Terceiro, houve poucos participantes das regiões nordeste, centro-oeste e nenhum da região norte, o que também não corresponde à distribuição da população brasileira. Estudos futuros devem investigar variações dos escores com amostras mais representativas do perfil nacional.

Os resultados do presente estudo indicam que a medida da EAD é válida e confiável para aplicação em contexto brasileiro. A estrutura de quatro fatores da versão brasileira da escala (Anexo 1) apresentou boas cargas fatoriais e bons índices de consistência interna tanto geral quanto dos fatores. A EAD se mostra um instrumento adequado para investigação de autoritarismo na população brasileira. Estudos futuros podem propor uma versão reduzida da escala uma vez que a literatura aponta que alfa de Cronbach maior do que 0,90 pode ser indicativo de itens redundantes (Tavakol \& Dennick, 2011). Estudos futuros envolvendo análise fatorial confirmatória, testagem da validade preditiva da medida e modelos explicativos utilizando a EAD também poderão ser feitos.

\section{Referências}

Adams, K. A., Nagoshi, C. T., Filip-Crawford, G., Terrell, H. K., \& Nagoshi, J. L. (2016). Components of gender-nonconformity Prejudice. International Journal of Transgenderism, 17(3-4), 185-198. doi: 10.1080/15532739.2016.1200509

Adorno, T., Frenkel-Brunswick, E., Levinson, D., \& Sanford, N. (1950). The Authoritarian Personality. New York: Harper.

Altemeyer, B. (1981). Right-Wing Authoritarianism. Winnipeg, Canada: University of Manitoba Press.

Altemeyer, B. (1988). Enemies of Freedom: Understanding Right-Wing Authoritarianism. San Francisco, CA: Jossey-Bass.

Altemeyer, B. (1996). The Authoritarian Specter. Cambridge, MA: Harvard University Press.

Altemeyer, B. (1998). The other "authoritarian personality." In M. Zanna (Ed.), Advances in experimental social psychology (Vol. 30, pp. 47-92). San Diego, CA: Academic Press.
Arendt, H. (1975). Elemente und Ursprünge totaler Herrschaft [As origens do totalitarismo]. New York: Harcourt.

Barros, T. S., Torres, A. R. S., \& Pereira, C. (2009). Autoritarismo e adesão a sistemas de valores psicossociais. Psico-USF, 14(1), 47-57. doi: 10.1590/S1413-82712009000100006

Benjamin, A. J., Jr. (2016). Right-Wing Authoritarianism and Attitudes toward Torture. Social Behavior and Personality, 44(6), 881-888. doi: 10.2224/sbp.2016.44.6.881

Borsa, J. C., Damásio, B. F., \& Bandeira, D. R. (2012). Adaptação e validação de instrumentos psicológicos entre culturas: algumas considerações. Paidéia (Ribeirão Preto), 22(53), 423-432. doi: 10.1590/S0103-863X2012000300014

Cantal, C., Milfont, T. L., Wilson, M. S., \& Gouveia, V. V. (2015). Differential effects of Right-Wing Authoritarianism and social dominance orientation on dimensions of generalized prejudice in Brazil. European Journal of Personality, 29, 1727. doi: 10.1002/per.1978

Carrara, S. (2012). Políticas e Direitos Sexuais no Brasil Contemporâneo. Bagoas-Estudos Gays: Gêneros e Sexualidades, 4(5), 132-148.

Carvacho, H., Zick, A., Haye, A., González, R., Manzi, J., Kocik, C., \& Bertl, M. (2013). On the relation between social class and prejudice: The roles of education, income, and ideological attitudes. European Journal of Social Psychology, 43, 272-285. doi: 10.1002/ejsp.1961

Cerutti, F., Ramos, S. P., \& Argimon, I. I. L. (2015). A implicação das atitudes parentais no uso de drogas na adolescência. Acta Colombiana de Psicología, 18(2), 173-181. doi: 10.14718/ ACP.2015.18.2.15

Cherry, F., \& Byrne, D. (1977). Authoritarianism. In T. Blass (Ed.), Personality variables in social behavior (pp. 109-133). Hillsdale, NJ: Erlbaum.

Costa, A. B., Bandeira, D. R., \& Nardi, H. C. (2015). Avaliação do preconceito contra diversidade sexual e de gênero: Construção de um instrumento. Estudos de Psicologia (Campinas), 32(2), 163172. doi: 10.1590/0103-166X2015000200002

Costa, A. B., Machado, W. L., Bandeira, D. R., \& Nardi, H. C. (2016). Validation Study of the Revised Version of the Scale of Prejudice Against Sexual and Gender Diversity in Brazil. Journal of Homosexuality, 63(11), 1446-1463. doi: 


\section{$10.1080 / 00918369.2016 .1222829$}

Coutinho, J. P. (2014). As ideias conservadoras. São Paulo, SP: Três Estrelas.

Crawford, J. T., Brandt, M. J., Inbar, Y., \& Mallinas, S. R. (2016). Right-Wing Authoritarianism predicts Prejudice Equally Toward "Gay Men and Lesbians" and "Homosexuals". Journal of Personality and Social Psychology, 111(2), e31-e45. doi: 10.1037/pspp0000070

Damásio, B. F. (2012). Uso da Análise Fatorial Exploratória em Psicologia. Avaliação Psicológica, 11(2), 213-228.

Datafolha. (2014). Intenção de voto para presidente da república. Recuperado em http://media.folha. uol.com.br/datafolha/2014/09/08/matriz-direita-x-esquerda.pdf

De Regt, S., Mortelmans, S., \& Smits, T. (2011). Left-wing authoritarianism is not a myth, but a worrisome reality. Evidence from 13 Eastern European countries. Communist and Post-Communist Studies, 44, 299-308. doi: 10.1016/j.postcomstud.2011.10.006

Duckitt, J. (1993). Right-Wing Authoritarianism among White South African Students: Its measurement and correlates. The Journal of Social Psychology, 133(4), 553-563. doi: 10.1080/00224545.1993.9712181

Duckitt, J., Bizumic, B., Krauss, S. W., \& Heled, E. (2010). A Tripartite Approach to Right-Wing Authoritarianism: The Authoritarianism-Conservatism-Traditionalism Model. Political Psychology, 31(5), 685-715. doi: 10.1111/j.1467-9221.2010.00781.x

Duckitt, J., \& Fisher, K. (2003). The impact of social threat on worldview and ideological attitudes. Political Psychology, 24, 199-222. doi: 10.1111/0162-895X.00322

Duckitt, J., Wagner, C., du Plessis, I., \& Birum, I. (2002). The Psychological Bases of Ideology and Prejudice: Testing a Dual Process Model. Journal of Personality and Social Psychology, 83(1), 75-93. doi: 10.1037//0022-3514.83.1.75

Etchezahar, E. (2012). Las Dimensiones del Autoritarismo: análisis de la Escala de Autoritarismo del Ala de Derechas (RWA) en una Muestra de Estudiantes Universitarios de la Ciudad de Buenos Aires. Psicologia Política, 12(25), 591-603.

Failla, Z. (2016). Retratos da leitura no Brasil. Rio de Janeiro, RJ: Sextante.
Funke, F. (2005). The Dimensionality of Right-Wing Authoritarianism: Lessons from the Dilemma between Theory and Measurement. Political Psychology, 26, 195-218. doi: 10.1111/j.1467$-9221.2005 .00415 . \mathrm{x}$

Goodnight, B. L., Cook, S. L., Parrott, D. J., \& Peterson, J. L. (2014). Effects of Masculinity, Authoritarianism, and Prejudice on Antigay Aggression: A Path Analysis of Gender-Role Enforcement. Psychology of Men, \& Masculinity, 15(4), 437-444. doi: 10.1037/a0034565

Gray, D., \& Durrheim, K. (2006). The Validity and Reliability of Right-Wing Authoritarianism in South Africa. South African Journal of Psychology, 36(3), 500-520. doi: 10.1177/008124630603600305

Guimarães, J. G., Torres, A. R. R., \& de Faria, M. R. G. V. (2005). Democracia e violência policial: O caso da Polícia Militar. Psicologia em Estudo, 10(2), 263-271. doi: 10.1590/S141373722005000200013

Güldü, Ö. (2011). SAĞ KANAT YETKECİLİ̆̈̇ ÖLÇEĞİ: UYARLAMA ÇALIŞMASI. Ankyra: Ankara Üniversitesi Sosyal Bilimler Enstitüsü Dergisi, 2(2), 27-51. doi: 10.1501/sbeder_0000000033

Hall, C. S., Lindzey, G., \& Campbell, J. B. (2008). Teorias da Personalidade (5. ed.). Porto Alegre, RS: Artmed.

Kohn, P. (1974). The Authoritarianism-rebellion Scale: A balanced $\mathrm{F}$ scale with left-wing reversals. Sociometry, 35, 176-189. doi: 10.2307/2786557

Lee, R., \& Warr, P. (1969). The Development and Standardization of a balanced F Scale. Journal of General Psychology, 81, 109-129. doi: 10.1080/00221309.1969.9710776

Mavor, K. I., Louis, W. R., \& Sibley, C. G. (2010). A bias-corrected Exploratory and Confirmatory Factor Analysis of Right-Wing Authoritarianism: Support for a three-factor Structure. Personality and Individual Differences, 48, 28-33. doi: 10.1016/j.paid.2009.08.006

Milgram, S. (1963). Behavioral Study of Obedience. Journal of Abnormal and Social Psychology, 67, 371-378. doi: 10.1037/h0040525

O'Connor, B. P. (2000). SPSS and SAS Programs for determining the Number of Components using Parallel Analysis and Velicer's MAP Test. Behavior Research Methods, Instruments, \& Compu- 
ters, 32(3), 396-402. doi: 10.3758/BF03200807

Passini, S. (2015). Different Ways of being Authoritarian: The distinct Effects of Authoritarian Dimensions on Values and Prejudice. Political Psychology, 38(1), 73-86. doi: 10.1111/ pops. 12309

Santelli, J., Ott, M. A., Lyon, M., Rogers, J., Summers, D., \& Schleifer, R. (2006). Abstinence and abstinence-only education: A review of US policies and programs. Journal of Adolescent Health, 38(1), 72-81. doi: 10.1016/j.jadohealth.2005.10.006

Santos, L. C. O. (2015). Aspectos religiosos, educacionais e valorativos da intenção de voto (Dissertação de mestrado, Universidade Federal da Paraíba, João Pessoa, PB, Brasil).

Scruton, R. (2015). O que é Conservadorismo. São Paulo, SP: É Realizações.

Sibley, C. G., \& Duckitt, J. (2008). Personality and Prejudice: A Meta-Analysis and Theoretical Review. Personality and Social Psychology Review, 12(3), 248-279. doi: $10.1177 / 1088868308319226$

Sousa, C. P. G. (2016). A influência do autoritarismo e do locus de controlo nas atitudes homofóbicas (Dissertação de mestrado, Universidade Lusófona de Humanidade e Tecnologias, Lisboa, Portugal).

Stones, C. R. (2006). Antigay Prejudice among Heterosexual Males: Right-Wing Authoritarianism as a stronger predictor than Social-Dominance Orientation and Heterosexual Identity. Social Behavior and Personality, 34(9), 1137-1150. doi: 10.2224/sbp.2006.34.9.1137
Tavakol, M., \& Dennick, R. (2011). Making Sense of Cronbach's Alpha. International Journal of Medical Education, 2, 53-55. doi: 10.5116/ ijme. $4 \mathrm{dfb} .8 \mathrm{dfd}$

Tee, N., \& Hegarty, P. (2006). Predicting Opposition to the Civil Rights of Trans Persons in the United Kingdom. Journal of Community, \& Applied Social Psychology, 16, 70-80. doi: 10.1002/ casp.851

Torres, A. R. R., de Faria, M. R. G. V., Guimarães, J. G., \& Martignoni, T. V. L. (2007). Análise Psicossocial do Posicionamento de Adolescentes com relação à Violência Policial. Psicologia em Estudo, 12(2), 229-238. doi: 10.1590/S141373722007000200003

Vilanova, F., Beria, F.M., Costa, A.B., \& Koller, S.H. (2017). Deindividuation: From Le Bon to the Social Identity Model of Deindividuation Effects. Cogent Psychology, 4:1308104. DOI: 10.1080/23311908.2017.1308104

Vilela, M. O. (2012). A Personalidade Autoritária do Chão de Fábrica à Gerência: Um estudo aplicando a Escala RWA adaptada da Escala "F" de Adorno (Dissertação de mestrado, Pontifícia Universidade Católica de Minas Gerais, Belo Horizonte, MG, Brasil).
Recebido: 16/05/2017

$1^{a}$ revisão:20/05/2017

$2^{a}$ revisão: 13/08/2017

Aceite final: 16/08/2017

(cc)BY BY 1 O(s) autor(es), 2018. Acesso aberto. Este artigo está distribuído nos termos da Licença Internacional Creative Commons Atribuição 4.0 (http://creativecommons.org/licenses/by/4.0/), que permite o uso, distribuição e reprodução sem restrições em qualquer meio, desde que você dê crédito apropriado ao(s) autor(es) original(ais) e à fonte, fornecer um link para a licença Creative Commons e indicar se as alterações foram feitas. 


\section{Anexo 1 \\ Versão Brasileira da Escala de Autoritarismo de Direita}

Este questionário busca entender as suas opiniões sobre diversos temas sociais. Por favor, marque o quanto você concorda com as afirmações abaixo:

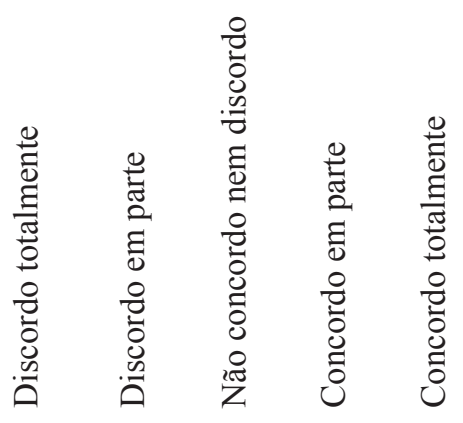

1 Do jeito que as coisas estão indo nesse país, serão necessárias medidas severas para endireitar os meliantes, os criminosos e os pervertidos. ${ }^{1}$

2 A situação do nosso país está ficando tão séria que ações firmes seriam justificadas se eliminassem os desordeiros e nos levassem de volta ao nosso verdadeiro caminho. ${ }^{1}$

3 Ser gentil com criminosos só os encoraja a tirar proveito de sua fraqueza, sendo melhor agir de maneira firme e dura com eles. ${ }^{1}$

4 A pena de morte é bárbara e nunca justificável. (R $)^{1}$

5 Os crimes e as desordens públicas recentes mostram que se quisermos preservar a lei e a ordem, devemos agir de forma mais dura com os desordeiros. ${ }^{1}$

6 O que o nosso país realmente precisa é uma dose forte e dura de lei e ordem. ${ }^{1}$

$7 \quad$ Nós deveríamos esmagar todos os elementos negativos que estão causando problemas na nossa sociedade. ${ }^{1}$

8 Nossas prisões são um desastre. Ao invés de tanta punição, os que estão em conflito com a lei merecem um cuidado muito melhor. $(\mathrm{R})^{1}$

9 Nós precisamos de maior tolerância e mais leniência no tratamento de infratores. $(\mathrm{R})^{1}$

10 As pessoas que dizem que nossas leis deveriam ser aplicadas de maneira mais rigorosa e severa estão erradas. $(R)^{1}$

11 Nossa sociedade NÃO precisa de um governo mais duro e leis mais rigorosas. $(\mathrm{R})^{1}$

12 Quanto maior o número de pessoas preparadas para desafiar o governo, melhor para a sociedade. ${ }^{2}$

13 Quanto maior o número de pessoas preparadas para protestar contra o governo, melhor para a sociedade. ${ }^{2}$

14 Quanto maior o número de pessoas preparadas para criticar as autoridades, melhor para a sociedade. ${ }^{2}$

15 As pessoas devem estar prontas para desafiar leis com as quais elas não concordam. ${ }^{2}$

16 Estudantes de colégios e universidades devem ser encorajados a desafiar, criticar e confrontar autoridades. ${ }^{2}$

$\begin{array}{lllll}1 & 2 & 3 & 4 & 5\end{array}$


17 É ótimo que atualmente muitos jovens estejam preparados para desafiar a autoridade. ${ }^{2}$

18 As pessoas deveriam ter as suas próprias preferências sexuais, mesmo se isso torná-las diferentes do resto da sociedade. $(\mathrm{R})^{3}$

19 Não há nada de errado com sexo antes do casamento. $(\mathrm{R})^{3}$

20 Não tem absolutamente nada errado com praias de nudismo. (R) ${ }^{3}$

21 As pessoas deveriam ter os seus próprios estilos de vida mesmo se isso torná-las diferentes do resto da sociedade. $(\mathrm{R})^{3}$

22 As pessoas deveriam ter as suas próprias crenças religiosas mesmo se isso torná-las diferentes do resto da sociedade. $(\mathrm{R})^{3}$

23 As pessoas deveriam prestar menos atenção à Bíblia e às outras formas antiquadas de orientação religiosa, e desenvolver seu próprio padrão do que é moral e imoral. $(\mathrm{R})^{3}$

24 As leis de Deus sobre aborto, pornografia e casamento devem ser seguidas à risca antes que seja tarde demais. ${ }^{3}$

25 Ninguém deveria se restringir ao que é moralmente correto. Ao invés disso, as pessoas deveriam se libertar e experimentar várias ideias e experiências diferentes. $(\mathrm{R})^{3}$

26 Tem muita coisa de errado com os valores, os costumes e a moralidade tradicionais. $(\mathrm{R})^{3}$

27 O segredo para uma boa vida é a obediência àqueles que estão no controle. $^{4}$

28 As autoridades devem ser obedecidas porque elas estão na melhor posição para saber o que é bom para o país. ${ }^{4}$

29 Nosso país será melhor se obedecermos nossos líderes. ${ }^{4}$

30 O segredo para uma boa vida é o respeito pela autoridade. ${ }^{4}$

31 O que nosso país mais precisa é disciplina, com todos seguindo nossos líderes. ${ }^{4}$

32 Nossos líderes deveriam ser obedecidos sem questionamento. ${ }^{4}$

33 As virtudes mais importantes que as crianças devem aprender são obediência e respeito à autoridade. ${ }^{4}$

34 Nosso país será melhor se mostrarmos respeito à autoridade. ${ }^{4}$

\begin{tabular}{|c|c|c|c|}
\hline 1 & 2 & 3 & 4 \\
\hline 1 & 2 & 3 & 4 \\
\hline 1 & 2 & 3 & 4 \\
\hline 1 & 2 & 3 & 4 \\
\hline 1 & 2 & 3 & 4 \\
\hline 1 & 2 & 3 & 4 \\
\hline 1 & 2 & 3 & 4 \\
\hline 1 & 2 & 3 & 4 \\
\hline 1 & 2 & 3 & 4 \\
\hline 1 & 2 & 3 & 4 \\
\hline 1 & 2 & 3 & 4 \\
\hline 1 & 2 & 3 & 4 \\
\hline 1 & 2 & 3 & 4 \\
\hline 1 & 2 & 3 & 4 \\
\hline 1 & 2 & 3 & 4 \\
\hline 1 & 2 & 3 & 4 \\
\hline 1 & 2 & 3 & 4 \\
\hline 1 & 2 & 3 & 4 \\
\hline
\end{tabular}

(R): Item com pontuação a ser recodificada para cálculo do escore do fator (i.e., respostas 1 neste item deve ser recodificadas como 5, e vice-versa, e respostas 2 neste item devem ser recodificadas como 4, e vice-versa). Após recodificação, os escores dos subfatores e o escore geral são calculados através de média aritmética simples.

': Item correspondente ao fator 'Autoritarismo'

2: Item correspondente ao fator 'Contestação à Autoridade'

3: Item correspondente ao fator 'Tradicionalismo'

4: Item correspondente ao fator 'Submissão à Autoridade'. 